\title{
The association of the serum levels of myostatin, follistatin, and interleukin- 6 with sarcopenia, and their impacts on survival in patients with hepatocellular carcinoma
}

Kanghyug Choi ${ }^{1, *}$, Hee Yoon Jang, ${ }^{1, *}$, Joong Mo Ahn², Sung Ho Hwang ${ }^{3}$, Jung Wha Chung ${ }^{4}$, Yun Suk Choi', Jin-Wook Kim', Eun Sun Jang', Gwang Hyeon Choi', and Sook-Hyang Jeong ${ }^{1}$

Departments of 1 Internal Medicine and ${ }^{2}$ Radiology, Seoul National University Bundang Hospital, Seoul National University College of Medicine, Seongnam; ${ }^{3}$ Department of Nursing, Daewon Univeristy College, Jecheon; ${ }^{4}$ Department of Internal Medicine, Wonkwang University Sanbon Hospital, Sanbon, Korea

\section{Graphical Abstract}

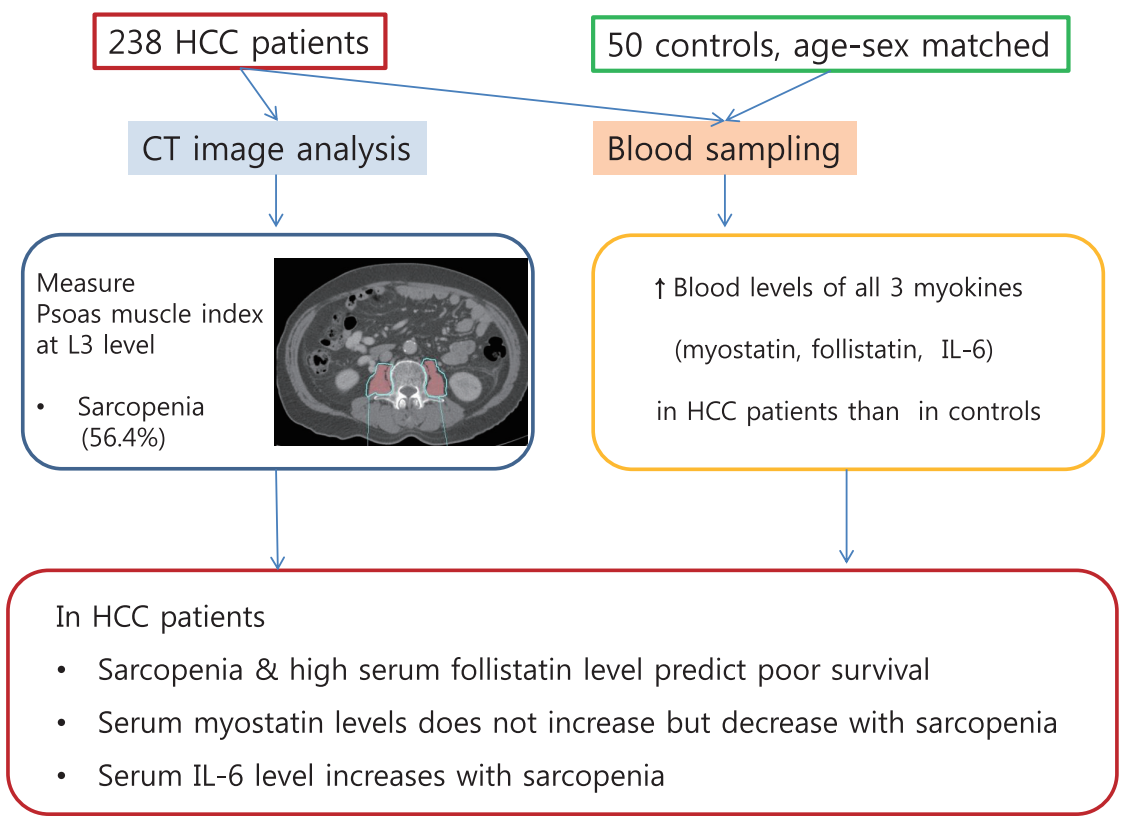

\section{Abbreviations:}

AFP, alpha-fetoprotein; AST, aspartate aminotransferase; BCLC, Barcelona Clinic Liver Cancer; BIA, bioelectrical impedance analysis; BMI, body mass index; $\mathrm{BMPs}$, bone morphogenetic protein; $\mathrm{Cl}$, confidence interval; $\mathrm{CT}$, computed tomography; HBV, hepatitis B virus; HCC, hepatocellular carcinoma; HR, hazard ratio; IL-6, interleukin-6; IRB, Institutional Review Board; MELD, model for end stage liver disease; MRI, magnetic resonance imaging; PMI, psoas muscle index; SD, standard deviations; TACE, transarterial chemoembolization; TGF-beta, transforming growth factor beta
Corresponding author: Sook-Hyang Jeong

Department of Internal Medicine, Seoul National University Bundang Hospital, Seoul National University College of Medicine, 82 Gumi-ro 173beon-gil, Bundang-gu, Seongnam 13620, Korea

Tel: +82-31-787-7029, Fax: +82-31-787-4052

E-mail: jsh@snubh.org

https://orcid.org/0000-0002-4916-7990

*These authors are equally contributed to this work. 
Background/Aims: The role of serum myokine levels in sarcopenia and the outcome of hepatocellular carcinoma (HCC) patients are not clear. This study investigated the serum levels of myostatin, follistatin, and interleukin-6 (IL-6) in HCC patients and their association with sarcopenia and survival.

Methods: Using prospectively collected pretreatment samples from 238 HCC patients in a hospital from 2012 to 2015, the serum levels of 3 myokines were determined and compared to 50 samples from age and sex-matched healthy controls. Sarcopenia was evaluated using the psoas muscle index (PMI) measured at the third lumbar level in the computed tomography, and clinical data were collected until 2017.

Results: The median levels of the 3 myokines for the male and female HCC patients were as follow: myostatin $(3,979.3$ and 2,976.3 pg/mL), follistatin $(2,118.5$ and $2,174.6 \mathrm{pg} / \mathrm{mL})$, and IL-6 (2.5 and $2.7 \mathrm{pg} / \mathrm{mL})$, respectively. Those in the HCC patients were all significantly higher than in the healthy controls. In the HCC patient, the median PMI was 4.43 (males) and $2.17 \mathrm{~cm}^{2} / \mathrm{m}^{2}$ (females) with a sarcopenic prevalence of $56.4 \%$. The serum levels of myostatin, IL- 6 and follistatin in the HCC patients showed a positive, negative, and no correlation with PMI, respectively. The serum follistatin level was an independent factor for poor survival in HCC patients.

Conclusions: The serum levels of myostatin, follistatin, and IL-6 and their correlation with sarcopenia and survival were presented in $\mathrm{HCC}$ patients for the first time. The role of the serum follistatin level as a poor prognostic biomarker warrants further study. (Clin Mol Hepatol 2020;26:492-505)

Keywords: Hepatocellular carcinoma; Myostatin; Follistatin; Interleukin 6; Sarcopenia

\footnotetext{
Study Highlights

- The association between the serum levels of myostatin, follistatin, and IL- 6 and PMI in HCC patients were presented for the first time.

- The median levels of the 3 myokines for the male and female HCC patients were all significantly higher than in the healthy controls.

- The serum levels of myostatin, LL-6 and follistatin in the HCC patients showed a positive, negative, and no correlation with PMI, respectively.

- The serum follistatin level was an independent factor for poor survival in HCC patients.
}

\section{INTRODUCTION}

Sarcopenia, defined as a decline in skeletal muscle mass and strength, is increasingly important in the aging society. Currently, the measurement of appendicular lean mass using bioelectrical impedance analysis (BIA) or dual energy $\mathrm{X}$-ray absorptiometry is the standard diagnostic modality of sarcopenia, which is stratified by gender. Evaluation of hand grip strength or gait speed provides additional accuracy for the diagnosis of sarcopenia. ${ }^{1,2}$

However, diagnosis of sarcopenia is not simple. The cut-off for those diagnostic criteria varies according to the race or country because of different physiques. Usually, the cut-off value has been set as below 2 standard deviations (SD) from the mean for young adults or as being in the bottom quintile, resulting in different cut-off values among various populations., ${ }^{3,4}$ Moreover, BIA may not be accurate for the diagnosis of sarcopenia in patients with chronic liver disease with ascites or edema, such as liver cirrhosis, hepatocellular carcinoma (HCC), and liver transplantation. ${ }^{5,6}$ In those patients, diagnosis of sarcopenia is important because of its deleterious role in the outcomes, which can be intervened upon by reinforcement of nutrition and exercise. ${ }^{\text {? }}$

Aside from the measurement of muscle mass and muscle strength, several serum biomarkers related to sarcopenia are being developed. There are many adipomyokines, secreted by skeletal myocytes or adipocytes, including muscle growth inhibitors (interleukin-6 [IL-6], myostatin, activins A/B, growth and differentiation factor 15) and muscle growth promoters (follistatin, irisin, bone morphogenetic protein [BMPs], and brain-derived neurotrophic factor). ${ }^{8-11}$ A recent study from Japan suggested the use of the serum myostatin level as a potential biomarker, showing that a high myostatin level was associated with sarcopenia and a reduced survival rate in liver cirrhosis patients. ${ }^{12}$

However, there have been no studies that have measured the serum levels of those adipomyokines among HCC patients, although sarcopenia is an independent factor for poor survival in HCC regardless of the stage of the tumor, degree of liver function, or cirrhotic status. ${ }^{7}$ The aim of this study was to investigate the serum levels of myostatin, follistatin, and IL-6, and to evaluate 
their association with sarcopenia and survival in HCC patients.

\section{PATIENTS AND METHODS}

\section{HCC patients and healthy controls}

We prospectively enrolled 258 newly diagnosed HCC patients at Seoul National University Bundang Hospital from January 2012 to December 2015, and followed them up until December 2017. After the exclusion of 20 patients whose serum samples were not available, 238 patients with HCC were the final subjects of this study. HCC was diagnosed according to histological findings or typical imaging characteristics as defined by the Korean Liver Cancer Study Group and National Cancer Center guidelines, which are similar to international guidelines. ${ }^{13}$ All of the subjects provided written informed consent, and this study was approved by the Institutional Review Board (IRB) of Seoul National University Bundang Hospital (B-1810-497-301). All methods were performed in accordance with national guidelines and regulation. Plasma samples from 50 healthy controls, which were matched for age and sex to the HCC patients, were obtained from a repository that included anonymized clinical data after IRB approval (B-1307/210-006).

\section{Clinical data collection and follow up of the subjects}

The clinical data at the diagnosis of HCC were entered into a predefined electronic case report form, which included age, sex, height, weight, alcohol intake, smoking history, performance status, etiology of HCC, comorbidities, blood levels of alpha-fetoprotein (AFP), albumin, serum aspartate aminotransferase (AST), alanine aminotransferase, total bilirubin, prothrombin time expressed as international normalized ratio, platelet count, creatinine, sodium, Child-Pugh score and class, model for end stage liver disease (MELD) score, tumor size, number, vascular invasion, TNM stage based on American Joint Committee on Cancer, 7th edition, ${ }^{14}$ and Barcelona Clinic Liver Cancer (BCLC) stage. ${ }^{15}$

The patients were prospectively followed for a median duration of 31.8 months until December 2017. The assessment of tumor response after treatment followed the modified Response Evaluation Criteria in Solid Tumors criteria using dynamic contrast enhanced computed tomography (CT) or magnetic resonance imaging (MRI), and complete remission was defined as disappearance of any intratumoral arterial enhancement in all target lesions. Recurrence of HCC was defined as the development of any new lesions or a new enhancing portion in the treated target lesion, and confirmed by medical records and CT or MRI images. The death of each patient was confirmed by a request to the Statistics Korea, and the cause of death was confirmed through a review of the medical records.

\section{Measurement of the psoas muscle area, calculation of the psoas muscle index (PMI) and determination of the cut-off for sarcopenia in HCC patients}

Multiphase CT scans were performed within 60 days of the diagnosis. We used a multidetector-row CT machine (Brilliance 64 or iCT256; Philips Medical Systems, Cleveland, OH, USA) for contrast enhancement CT examinations. Using a power injector (Stellant D, Medrad, Indianola, PA, USA), an intravenous non-ionic contrast material (2 mL/kg, iomeprol; $350 \mathrm{mg}$ iodine $/ \mathrm{mL}$, lomeron 350; Bracco, Milano, Italy) was administered through the antecubital vein.

The 2-dimensional cross-sectional area of the bilateral psoas muscles on the pre-contrast enhanced phase image was measured at the middle level of the third lumbar vertebra (L3). We used the Rapidia 3D software (v2.8; INFINITT Healthcare, Seoul, Korea) to outline the psoas muscle boundary. The borders of the psoas muscles were manually outlined at a setting from 31 to 100 Hounsfield Units to exclude more strictly the vasculature and areas of fatty infiltration. ${ }^{16-19}$ The measured psoas muscle area was divided by the patient height squared for the calculation of the PMI $\left(\mathrm{cm}^{2} / \mathrm{m}^{2}\right)$. For the measurement of the PMI, one fellow researcher (HYJ) and one research nurse (SHH) independently measured the same group of $\mathrm{CT}$ images from 20 randomly selected patients and compared the results. The interobserver agreement was $97 \%$, and then, the research nurse completed the measurement of the whole series of $\mathrm{CT}$ images from the subjects.

To determine the sex-specific cut-offs of the PMI for defining sarcopenia, we used the R software package (version 3.3.2; R Foundation for statistical Computing, Vienna, Austria; http:// www.R-project.org/) for optimum stratification which found the most significant value by using the log-rank chi-square statistic. ${ }^{20,21}$ The cut-off values for the PMI were set at $4.98 \mathrm{~cm}^{2} / \mathrm{m}^{2}$ for male patients, and $1.17 \mathrm{~cm}^{2} / \mathrm{m}^{2}$ for female patients.

\section{Blood sample storage and measurement of the serum levels of myostatin, follistatin, and IL- 6}

Blood samples were collected from the HCC patients before 
treatment, and serum aliquots were stored at $-70^{\circ} \mathrm{C}$ until the time of the measurement. Serum or plasma levels of myostatin, follistatin and IL-6 in the healthy controls and HCC patients were assessed using the commercial Quantikine ELISA Immunoassays (R\&D Systems, Inc., Minneapolis, MN, USA) following the manufacturer's instructions and done in duplicates by an experienced researcher (YSC).

\section{Statistical analysis}

The data were presented as the mean $\pm S D$ or the median with the interquartile range for continuous variables, and as a number and percentage for categorical variables. Student's independent $t$ test (continuous parametric variables) and Pearson's chi-square (categorical variables) were used to compare the two groups. A two-tailed $P$-value of $<0.05$ was considered statistically significant. Spearman's rank correlation was used to find the correlation between the PMI and the level of myostatin, follistatin and IL-6,

Table 1. Clinical characteristics of 238 patients with hepatocellular carcinoma and 50 healthy controls

\begin{tabular}{|c|c|c|}
\hline Variable & HCC patients $(n=238)$ & Healthy controls $(n=50)$ \\
\hline Age (years) & $59(53-69)$ & $64(53-71)$ \\
\hline$\geq 65$ & $93(39.1)$ & $16(32.0)$ \\
\hline Male & $193(81.1)$ & $40(80.0)$ \\
\hline Body mass index $\left(\mathrm{kg} / \mathrm{m}^{2}\right)$ & $23.7(21.8-25.8)$ & $23.5(21.7-25.4)$ \\
\hline$>25$ & $80(33.6)$ & $16(32.0)$ \\
\hline HBV/HCV/non-viral & $177 / 22 / 39$ (69.7/ 9.2/ 21.1) & \\
\hline Platelet $\left(10^{3} / \mu \mathrm{L}\right)$ & $131(92-184)$ & $228(161-296)$ \\
\hline Albumin (g/dL) & $4.0(3.5-4.3)$ & $4.5(4.3-4.6)$ \\
\hline Total bilirubin (mg/dL) & $0.8(0.5-1.1)$ & $1.0(0.8-1.3)$ \\
\hline AST (U/L) & $44(32-72)$ & $21(19-29)$ \\
\hline $\operatorname{ALT}(\mathrm{U} / \mathrm{L})$ & $37(23-55)$ & $21(18-27)$ \\
\hline Prothrombin time (INR) & $1.02(1.0-1.14)$ & $1.01(0.88-1.15)$ \\
\hline Sodium (mmol/L) & $138(136-140)$ & $142(139-144)$ \\
\hline Creatinine (mg/dL) & $0.8(0.67-0.96)$ & $1.1(1.0-1.2)$ \\
\hline Child-Pugh class A/B & $204(85.7) / 34(14.3)$ & \\
\hline MELD score & $4(2-6)$ & \\
\hline Presence of cirrhosis & $171(71.8)$ & \\
\hline Alpha-fetoprotein (ng/mL) & $21.7(4.0-837.4)$ & \\
\hline Tumor size (cm) & $3.5(2-7.3)$ & \\
\hline TNM stage I/II/III/IV & $111(46.6) / 43(18.1) / 80(33.6) / 4(1.7)$ & \\
\hline $\mathrm{BCLC}$ stage $0 / \mathrm{A} / \mathrm{B} / \mathrm{C}$ & $43(18.1) / 95$ (39.9)/43 (18.1)/57 (23.9) & \\
\hline \multicolumn{3}{|l|}{ Initial therapy } \\
\hline Resection/RFA & $8(3.4) / 38(16.0)$ & \\
\hline TACE/systemic therapy & $187(78.6) / 3(1.3)$ & \\
\hline Follow-up (months) & $31.9 \pm 9.2$ & \\
\hline Number of deaths & $45(18.9)$ & \\
\hline Recurrence free survival (months) & $19.5(0.7-66.4)$ & \\
\hline Overall survival (months) & $31.9(1.1-67.9)$ & \\
\hline
\end{tabular}

Values are presented as median (interquartile range), mean \pm standard deviation, or number (\%).

HCC, hepatocellular carcinoma; HBV, hepatitis B virus; HCV, hepatitis C virus; AST, aspartate aminotransferase; ALT, alanine aminotransferase; INR, international normalized ratio; MELD, model for end-stage liver disease; BCLC, Barcelona Clinic Liver Cancer; RFA, radiofrequency ablation; TACE, transarterial chemoembolization. 
and the coefficient of the correlation was indicated as Spearman's rho ( $\rho)$.

Overall survival and recurrence-free survival were analyzed with Kaplan-Meier curves, and the log-rank test was used to compare the survival between the two groups. To determine the independent factors for the overall survival of the HCC patients, univariate and multivariate analyses were performed. For the multivariable analyses using the Cox proportional hazard model, we put the variables, that had $P<0.1$ for the correlation with mortality in the univariable analysis and selected the most fitted final multivariable model. All statistical analyses were performed using SPSS Statistics (version 22.0; SPSS Inc., Chicago, IL, USA).

\section{RESULTS}

\section{Baseline characteristics of the HCC patients and healthy controls}

The baseline characteristics of the 238 HCC patients and 50 healthy controls are summarized in Table 1. There were no missing data for any variables among the HCC patients in Table 1. The HCC patients had a median age of 59 years; $81 \%$ were male; $70 \%$ had hepatitis B virus (HBV) surface antigen positivity, and the median body mass index (BMI) was $23.7 \mathrm{~kg} / \mathrm{m}^{2}$. The ChildPugh class was A in $85.7 \%$ and B in $14.3 \%$, and there was no class $C$ in this study. The median tumor size was $3.5 \mathrm{~cm}$ in diameter, and the BCLC stage $0, A, B$, and $C$ were $18.1 \%, 39.9 \%$, $18.1 \%$, and $23.9 \%$, respectively. The high proportion of HCC patients receiving transarterial chemoembolization (TACE) could be explained as the availability of superselective TACE experts in this center, and as such, physicians and patients tended to prefer TACE to resection as the first treatment with curative intention, especially in the case of old age, marginal liver function or more than one lesion number.

During a median follow-up of 31.9 months, 45 patients died (18.9\%). The median overall survival was 31.9 months (range, 1.1-67.9), and the median recurrence-free survival was 19.5 months (range, 0.7-66.4).

The 50 plasma samples were obtained from a remote repository of health check examinees showing normal laboratory results and diagnosed as healthy persons; their median age was 64 years, and the male proportion was $80 \%$.

\section{Serum levels of myostatin, follistatin and IL- 6 and their relationship with the PMI according to gender in the HCC patients}

The median serum levels of myostatin, follistatin and IL- 6 in the 50 healthy controls $(3,150.8,1,248.4$, and $0.81 \mathrm{pg} / \mathrm{mL}$, respectively) and $238 \mathrm{HCC}$ patients $(3,816.3,2,131.2$, and $2.52 \mathrm{pg} / \mathrm{mL}$, respectively) are summarized in Table 2 . The serum levels of the above 3 cytokines were all significantly higher in the HCC patients than in the healthy controls. Moreover, the serum median levels of myostatin were higher in the male $(3,979.3 \mathrm{pg} / \mathrm{mL})$ than in the female HCC patients $(2,976.3 \mathrm{pg} / \mathrm{mL})(P<0.001)$, while both the follistatin and IL- 6 levels were not significantly different between the male and female HCC patients. All the serum levels of the 3 myokines of the HCC patients and healthy controls are plotted in Supplementary Figure 1.

Table 2. Serum levels of myostatin, follistatin, IL-6, and psoas muscle index in the healthy control group and patients with hepatocellular carcinoma

\begin{tabular}{|c|c|c|c|c|}
\hline \multirow{2}{*}{ Variable } & \multirow{2}{*}{ Healthy control $(n=50)$} & \multicolumn{3}{|c|}{ HCC patients $(n=238)$} \\
\hline & & Total $(n=238)$ & Male $(n=193)$ & Female $(n=45)$ \\
\hline Myostatin (pg/mL) & $\begin{array}{c}3,150.8^{*} \\
(1,168.3-8,916.4)\end{array}$ & $\begin{array}{c}3,816.3 \\
(2,612.5-5,395.1)\end{array}$ & $\begin{array}{c}3,979.3 \\
(2,768.7-5,679.8)\end{array}$ & $\begin{array}{c}2,976.3 \\
(1,963.2-3,901.4)\end{array}$ \\
\hline Follistatin (pg/mL) & $\begin{array}{c}1,248.4^{*} \\
(473.9-2,637.0)\end{array}$ & $\begin{array}{c}2,131.2 \\
(1,572.3-2,886.1)\end{array}$ & $\begin{array}{c}2,118.5 \\
(1,554.6-2,964.7)\end{array}$ & $\begin{array}{c}2,174.6 \\
(1,697.9-2,760.3)\end{array}$ \\
\hline IL-6 (pg/mL) & $\begin{array}{c}0.81^{\dagger} \\
(0.06-5.75)\end{array}$ & $\begin{array}{c}2.52 \\
(1.40-4.99)\end{array}$ & $\begin{array}{c}2.50 \\
(1.41-4.86)\end{array}$ & $\begin{array}{c}2.75 \\
(1.38-5.05)\end{array}$ \\
\hline PMI $\left(\mathrm{cm}^{2} / \mathrm{m}^{2}\right)$ & & $\begin{array}{c}4.12 \\
(2.98-5.04)\end{array}$ & $\begin{array}{c}4.43 \\
(3.54-5.31)\end{array}$ & $\begin{array}{c}2.17 \\
(1.64-2.67)\end{array}$ \\
\hline
\end{tabular}

Values are presented as median (interquartile range).

IL-6, interleukin-6; HCC, hepatocellular carcinoma; PMI, psoas muscle index.

${ }^{*} P<0.001$ vs. patients with HCC.

${ }^{\dagger} P<0.05$ vs. patients with HCC. 
The median level of PMI was $4.43 \mathrm{~cm}^{2} / \mathrm{m}^{2}$ in the male and 2.17 $\mathrm{cm}^{2} / \mathrm{m}^{2}$ in the female HCC patients (Table 2). According to the optimized cut-off of $4.98 \mathrm{~cm}^{2} / \mathrm{m}^{2}$ for male patients and $1.17 \mathrm{~cm}^{2} / \mathrm{m}^{2}$ for female patients, the prevalence of sarcopenia was $56.4 \%$ overall (135/238), $67.4 \%$ in the male and $11.2 \%$ in the female patients, showing a remarkably high prevalence in the male HCC pa- tients. Compared to the non-sarcopenic group, the sarcopenic group had a higher proportion of male patients, a lower BMI, a higher proportion of smoking and larger tumor size (Table 3).

The relationship between myostatin, follistatin and IL-6 each with PMI is shown in Figure 1. The serum myostatin levels showed a positive correlation with the PMI $(\rho=0.356, P<0.001)$, suggest-

Table 3. Comparison of characteristics between sarcopenia and no sarcopenia group

\begin{tabular}{|c|c|c|c|}
\hline Variable & No Sarcopenia $(n=103)$ & Sarcopenia $(n=135)$ & $P$-value \\
\hline Age (years) & $58(50-67)$ & $68(59-77)$ & 0.177 \\
\hline$\geq 65$ & $37(35.9)$ & $56(41.5)$ & 0.384 \\
\hline Male sex & $63(61.2)$ & $130(96.3)$ & $<0.001$ \\
\hline Body mass index $\left(\mathrm{kg} / \mathrm{m}^{2}\right)$ & $24.1(22.0-26.1)$ & $22.6(20.6-24.5)$ & 0.004 \\
\hline Smoking & $16(15.5)$ & $36(26.7)$ & 0.039 \\
\hline Alcohol intake & $42(41.1)$ & $96(71.1)$ & 0.102 \\
\hline \multicolumn{4}{|l|}{ Etiology } \\
\hline HBV/HCV/non-viral & $76(73.8) / 13(12.6) / 14(13.6)$ & $91(67.4) / 10(7.4) / 34(25.2)$ & 0.090 \\
\hline Hypertension & $32(31.1)$ & $52(38.5)$ & 0.233 \\
\hline Diabetes & $23(22.3)$ & $37(27.4)$ & 0.371 \\
\hline Platelet $\left(10^{3} / \mu \mathrm{L}\right)$ & $130(89-178)$ & $139(95-195)$ & 0.002 \\
\hline Albumin (g/dL) & $4.0(3.5-4.3)$ & $3.9(3.2-4.1)$ & 0.883 \\
\hline Total bilirubin (mg/dL) & $0.8(0.5-1.1)$ & $0.8(0.5-1.2)$ & 0.187 \\
\hline AST (U/L) & $43(33-72)$ & $47(28-73)$ & 0.105 \\
\hline $\operatorname{ALT}(U / L)$ & $38(24-56)$ & $28(19-52)$ & 0.459 \\
\hline Prothrombin time (INR) & $1.01(1.00-1.13)$ & $1.02(1.00-1.14)$ & 0.822 \\
\hline Creatinine (mg/dL) & $0.81(0.68-0.96)$ & $0.75(0.61-0.94)$ & 0.193 \\
\hline Myostatin (pg/mL) & $3,990.1(2,824.5-5,630.1)$ & $2,785.9(1,963.2-4,446.9)$ & 0.077 \\
\hline Follistatin $(\mathrm{pg} / \mathrm{mL})$ & $2,124.2(1,551.0-2,903.0)$ & 2,139.6 (1,689.0-2,816.9) & 0.107 \\
\hline IL-6 (pg/mL) & $2.39(1.27-4.38)$ & $3.22(1.76-7.75)$ & 0.040 \\
\hline Child-Pugh score & $5(5-6)$ & $5(5-6)$ & 0.226 \\
\hline Child-Pugh class A/B & $91(88.3) / 12(11.7)$ & $113(83.7) / 22(16.3)$ & 0.310 \\
\hline MELD score & $4(2-6)$ & $4(1-6)$ & 0.201 \\
\hline MELD score $\geq 6$ & $73(70.9)$ & $95(70.4)$ & 0.933 \\
\hline Liver cirrhosis (\%) & $74(71.8)$ & $97(71.9)$ & 0.999 \\
\hline Alpha-fetoprotein (ng/mL) & $19.1(4.1-735)$ & $23.2(3-1,269)$ & 0.190 \\
\hline Tumor size $(\mathrm{cm})$ & $3.3(2.0-7.2)$ & $4.1(2.3-9.0)$ & $<0.001$ \\
\hline TNM stage $|/||/|||$ & $54(52.4) / 22(21.4) / 27(26.2)$ & $57(42.2) / 21(15.6) / 57(42.3)$ & 0.062 \\
\hline $\mathrm{BCLC}$ stage $0 / \mathrm{A} / \mathrm{B} / \mathrm{C}$ & $25(24.3) / 43(41.7) / 18(17.5) / 17(16.5)$ & $18(13.3) / 52(38.5) / 25(18.5) / 40(29.6)$ & 0.041 \\
\hline Psoas muscle index $\left(\mathrm{cm}^{2} / \mathrm{m}^{2}\right)$ & $4.6(3.8-5.4)$ & $2.4(1.8-2.9)$ & $<0.001$ \\
\hline Overall survival (months) & $32.3(12.7-43.6)$ & $29.6(8.7-48.6)$ & 0.076 \\
\hline Recurrence free survival (months) & $13.3(6.6-28.2)$ & $17.0(3.8-32.7)$ & 0.621 \\
\hline
\end{tabular}

Values are presented as median (interquartile range) or number (\%).

HBV, hepatitis B virus; HCV, hepatitis C virus; AST, aspartate aminotransferase; ALT, alanine aminotransferase; INR, international normalized ratio; IL-6, interleukin-6; MELD, model for end-stage liver disease; BCLC, Barcelona Clinic Liver Cancer. 


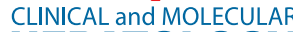

\section{HEPATOLOGY}

Volume_26 Number_4 October 2020

ing that the myostatin level increased with increasing muscle mass, though the correlation was weak. In contrast, the serum IL-6 levels showed a weakly negative correlation with the PMI ( $\rho=-0.174, P=0.009)$, such that the IL- 6 levels increased with progressive sarcopenia. The serum follistatin levels tended to be negatively correlated with the PMI ( $\rho=-0.124, P=0.055)$ with borderline statistical significance, but it was significantly correlated in female patients ( $\rho=-0.316, P=0.034$; Supplementary Table 1).

Among the 3 myokines, the IL- 6 levels showed a negative correlation with the myostatin $(\rho=-0.288, P<0.0001)$ and a positive correlation with the follistatin level $(\rho=0.422, P<0.0001)$. The serum myostatin level was negatively correlated with the follistatin level significantly ( $\rho=-0.218, P=0.0007$, Supplementary Fig. 2).

\section{Serum levels of myostatin, follistatin and IL- 6 and their relationship with liver function and tumor extent in HCC patients}

The serum levels of those 3 cytokines according to the liver function in terms of the Child-Pugh class and tumor stage are

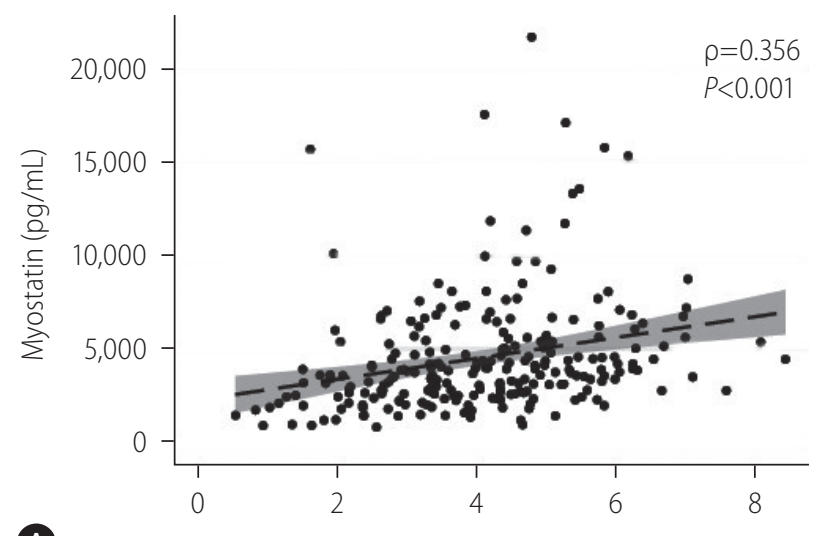

A

PMI

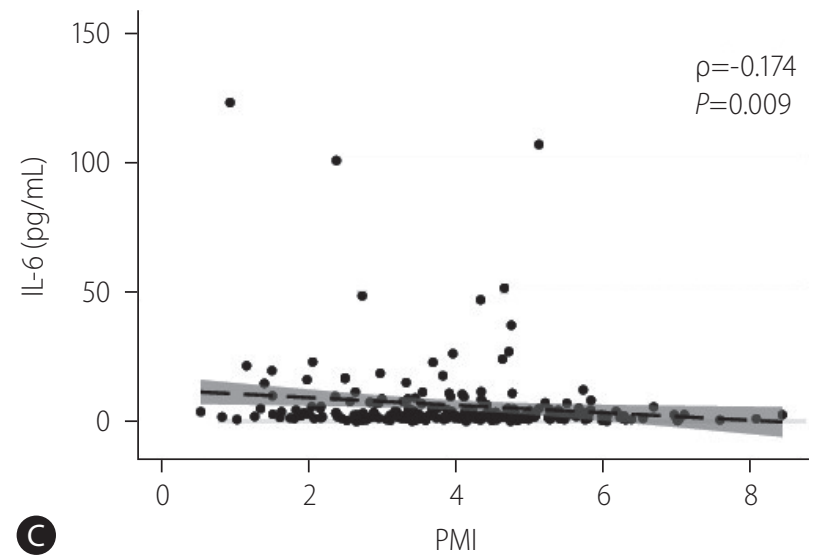

summarized in Figure 2. Compared to the patients with Child Pugh class $A$, the patients with Child Pugh class B had a significantly increased level of follistatin $(P=0.002)$, IL-6 $(P=0.002)$, and myostatin ( $P=0.006)$.

According to tumor progression by TNM stage or BCLC stage, the serum follistatin and IL-6 levels remarkably increased, especially for TNM stage 4 or BCLC stage C. However, the serum level of myostatin decreased in TNM stage 4 or BCLC stage $C$, showing that the trend was not clearly defined.

\section{Serum levels of myostatin, follistatin and IL-6 and their relationship with survival in HCC patients}

When the HCC patients were divided into two groups (highand low-level groups) based on the median serum level of myostatin, follistatin, and IL-6, the overall survival curves between the high- or low-level group are summarized in Figure 3. While the overall 5-year survival rate was not different between the high (74.8\%) and low myostatin (68.0\%) group $(P=0.062)$, the high follistatin group had a significantly lower 5 -year overall survival

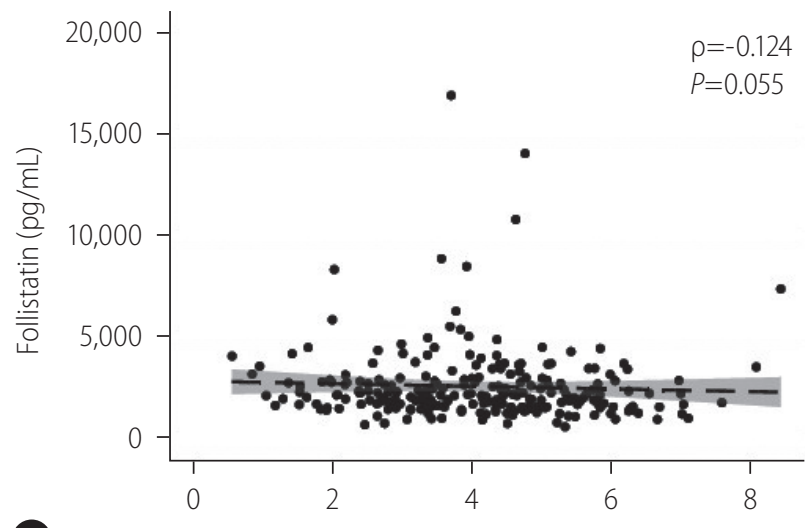

B

PMI

Figure 1. Relationship between the PMl and serum cytokine levels, (A) serum myostatin levels, (B) serum follistatin levels, (C) serum IL-6 levels. Spearman's rank correlation was used to find the correlation between the PMI and the level of myostatin, follistatin and IL-6, and the coefficient of the correlation was indicated as Spearman's rho ( $\rho)$. Serum myostatin levels showed a positive and linear correlation with PMI, though the correlation was weak ( $\rho=0.356, P<0.001$ ). In contrast, the serum IL-6 levels showed a negative association with the PMI, which was also a weak correlation $(\rho=-0.174, P=0.009)$. The serum follistatin levels were not correlated with the PMI. PMI, psoas muscle index; IL-6, interleukin-6. 
rate $(78.4 \%)$ than that of the low follistatin group (89.6\%, $P<0.001)$. Likewise, the high IL-6 group had a significantly shorter 5 -year overall survival rate $(78.4 \%)$ than that of the low IL-6 group $(85.8 \%, P=0.018)$.

For the recurrence-free survival, the high follistatin group showed a significantly poor survival than that of the low follistatin group, while the myostatin and IL-6 levels did not show a significant difference (Supplementary Fig. 3).

\section{Univariate and multivariate analyses of the parameters contributing to the overall survival}

The results of the univariable and multivariable analyses are summarized in Table 4. In the univariable analysis, current alcohol intake $(P=0.047)$, serum AFP $(P=0.033)$, albumin $(P=0.002)$, fol- listatin $(P=0.034)$, and IL-6 $(P=0.021)$ levels, MELD score $(P=0.005)$, ECOG performance stage $(P=0.001)$, TNM stage $(P<0.001)$, and presence of sarcopenia $(P=0.012)$ were significantly associated with the overall survival. The overall survival between sarcopenic group and non-sarcopenic group is shown in Figure 4.

Multivariable analysis showed that the follistatin level (hazard ratio $[\mathrm{HR}], 2.21 ; 95 \%$ confidence interval [CI], 1.13 to 4.30 ; $P=0.020)$ and the presence of sarcopenia (HR, 2.11; 95\% Cl, 1.07 to $4.17 ; P=0.031)$ remained statistically significant along with the MELD score (HR, 2.17; 95\% Cl, 1.17 to 4.01; $P=0.013)$, and TNM stage ( $\mathrm{HR}, 9.46 ; 95 \% \mathrm{Cl}, 3.33$ to $26.9 ; P<0.001)$. Therefore, the serum follistatin level was an independent factor associated with the overall survival. However, it was not independently associated with recurrence-free survival (data not shown).
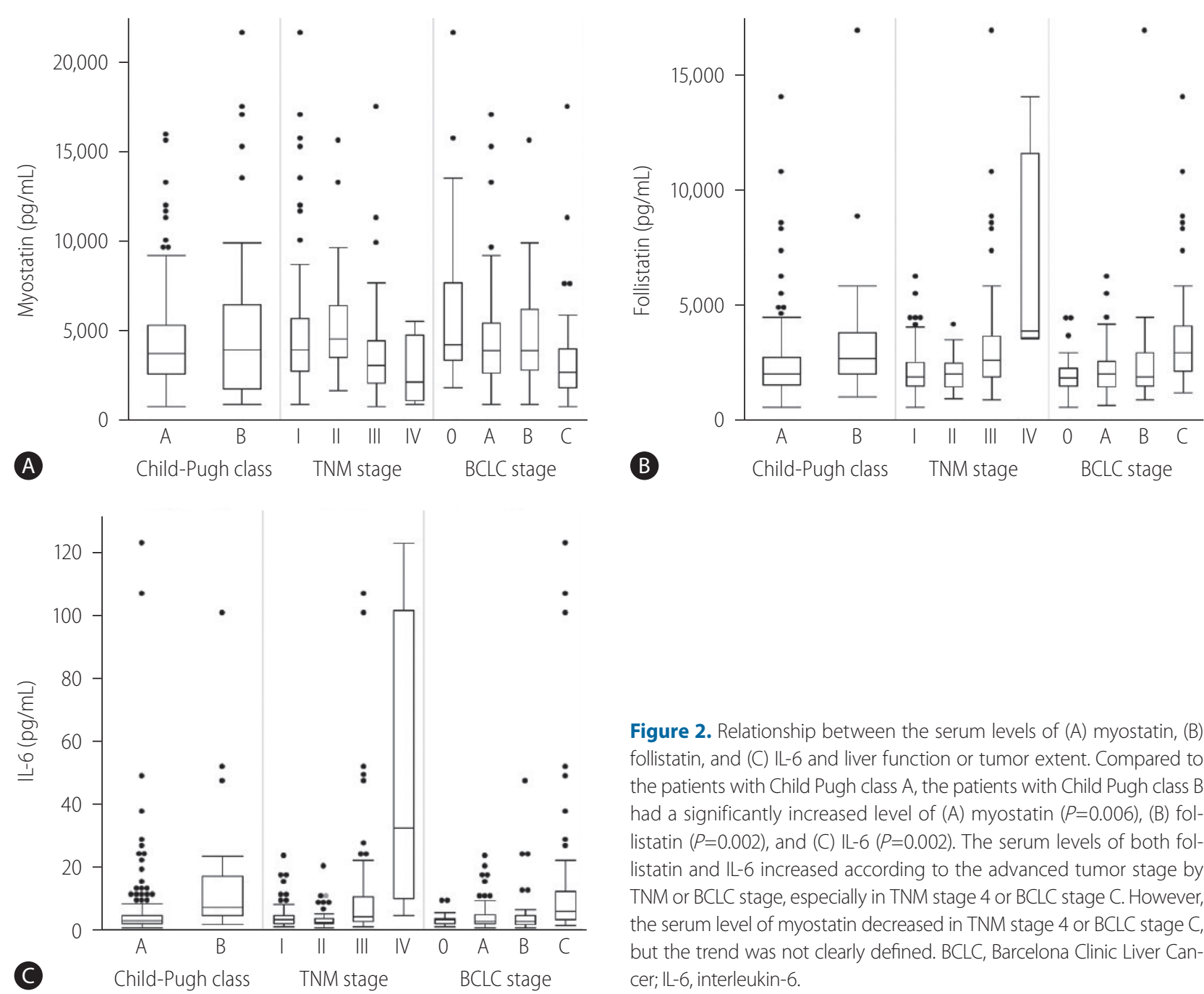

Figure 2. Relationship between the serum levels of (A) myostatin, (B) follistatin, and (C) IL-6 and liver function or tumor extent. Compared to the patients with Child Pugh class $A$, the patients with Child Pugh class B had a significantly increased level of $(A)$ myostatin $(P=0.006)$, (B) follistatin $(P=0.002)$, and $(C)$ IL-6 ( $P=0.002)$. The serum levels of both follistatin and IL-6 increased according to the advanced tumor stage by TNM or BCLC stage, especially in TNM stage 4 or BCLC stage C. However the serum level of myostatin decreased in TNM stage 4 or $B C L C$ stage $C$, but the trend was not clearly defined. BCLC, Barcelona Clinic Liver Cancer; lL-6, interleukin-6. 


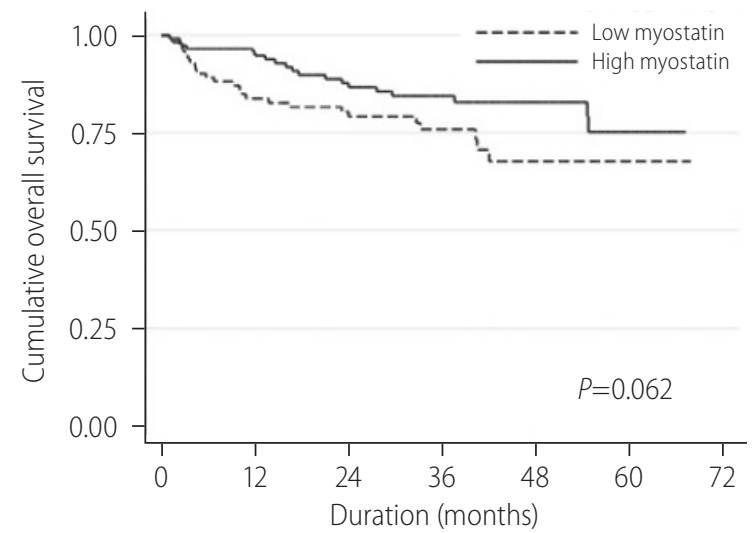

Number at risk

$\begin{array}{lllllll}\text { Low myostatin } 119 & 75 & 65 & 39 & 17 & 3 & 0\end{array}$ High myostatin $\begin{array}{lllllll}119 & 101 & 85 & 59 & 32 & 13 & 0\end{array}$

A

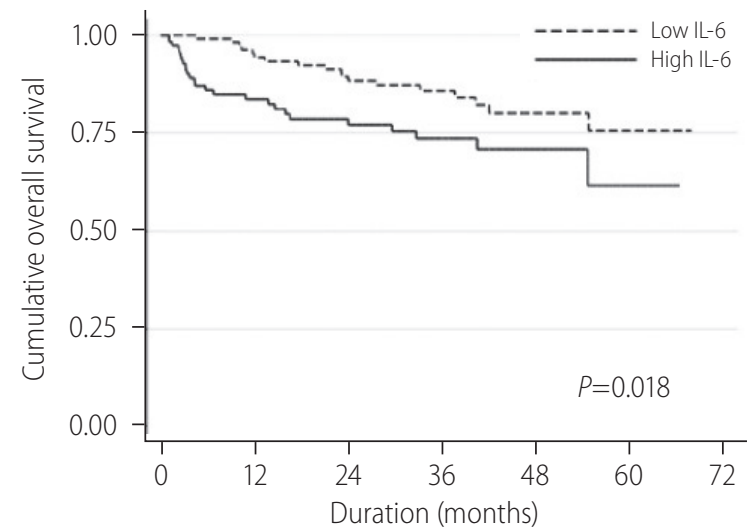

Number at risk

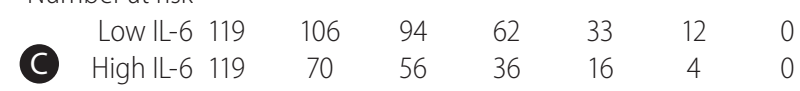

\section{DISCUSSION}

This study evaluated the serum levels of myostatin, follistatin and IL- 6 among HCC patients, and they all were significantly higher than in the healthy controls. The sarcopenic prevalence in terms of a low PMI using a gender-specific cut-off was $56 \%$ in the Korean HCC patients at our center, where HBV is the major cause of HCC. Unexpectedly, the serum myostatin levels increased with increasing muscle mass, while serum IL-6 levels increased with progressive sarcopenia, suggesting that myostatin may not have a role in inducing sarcopenia, while IL-6 did, and myostatin may be downregulated in response to sarcopenia in HCC patients. Both the follistatin and IL-6 levels increased with the tumor stage (TNM and BCLC staging systems), while the serum myostatin level did not. Interestingly, the serum follistatin level was an indepen-

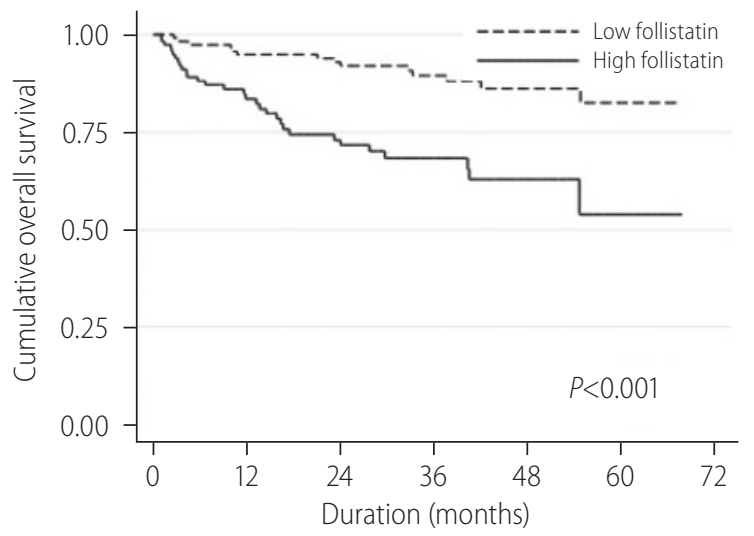

Number at risk

$\begin{array}{lllllll}\text { Low follistatin } 119 & 107 & 98 & 65 & 35 & 13 & 0\end{array}$ $\begin{array}{lllllll}\text { High follistatin } 119 & 69 & 52 & 33 & 14 & 3 & 0\end{array}$

B

Figure 3. Overall survival rate in regards to the serum levels of (A) myostatin, (B) follistatin, and (C) serum IL-6. In Kaplan-Meier survival analysis, the overall 5-year survival rate was not different between the high (74.8\%) and low myostatin (68.0\%) group ( $P=0.062)(A)$. However, the high follistatin group had a significantly shorter 5 -year overall survival rate $(78.4 \%)$ than that of the low follistatin group $(89.6 \%, P<0.001)$ (B). Likewise, the high IL-6 group had a significantly shorter 5 -year overall survival rate (78.4\%) than that of the low IL-6 group $(85.8 \%, P=0.018)$ (C). IL-6, interleukin-6.

dent factor for the overall survival of the HCC patients, while the myostatin or IL-6 level was not.

Myostatin is a member of the transforming growth factor beta (TGF-beta) family and the first known cytokine to be a negative regulator of muscles. ${ }^{22-24}$ In mice, an increased serum level of myostatin caused muscle atrophy, and a prolonged absence of myostatin reduces sarcopenia. However, a study that included 66 Scottish men showed that the serum myostatin level was not significantly different among young subjects, mildly sarcopenic and severely sarcopenic elderly subjects. Moreover, low serum myostatin levels were associated with a low skeletal muscle mass in healthy community-living older men, but not in women. ${ }^{25}$

In Japanese cirrhosis patients (median age of 68 years; $55 \%$ males; $62 \%$ Child-Pugh class A, 20\% HCC patients included), higher serum myostatin levels showed a negative correlation with 
Table 4. Univariate and multivariate analysis of factors related to the mortality

\begin{tabular}{|c|c|c|c|c|}
\hline \multirow{2}{*}{ Variable } & \multicolumn{2}{|c|}{ Univariate } & \multicolumn{2}{|c|}{ Multivariate } \\
\hline & HR (95\% Cl) & $P$-value & $\mathrm{HR}(95 \% \mathrm{Cl})$ & $P$-value \\
\hline \multicolumn{5}{|l|}{ Age (years) } \\
\hline$\geq 65(n=93)$ & $0.62(0.33-1.19)$ & 0.151 & & \\
\hline \multicolumn{5}{|l|}{ Sex } \\
\hline Male ( $n=193)$ & $1.37(0.58-3.25)$ & 0.469 & & \\
\hline \multicolumn{5}{|l|}{$\mathrm{BMI}\left(\mathrm{kg} / \mathrm{m}^{2}\right)$} \\
\hline$\geq 25(n=80)$ & $0.81(0.43-1.53)$ & 0.524 & & \\
\hline \multicolumn{5}{|l|}{ Alcoholics } \\
\hline Yes $(n=48)$ & $1.92(1.01-3.67)$ & 0.047 & & \\
\hline \multicolumn{5}{|l|}{ Smoking PY } \\
\hline$\geq 10 \mathrm{PY}(\mathrm{n}=104)$ & $1.06(0.59-1.90)$ & 0.839 & & \\
\hline \multicolumn{5}{|l|}{ Etiology } \\
\hline Viral $(n=191)$ & $0.87(0.42-1.82)$ & 0.716 & & \\
\hline \multicolumn{5}{|l|}{ AFP (ng/mL) } \\
\hline$\geq 20(n=121)$ & $4.5(2.2-9.13)$ & $<0.001$ & $2.09(0.99-4.45)$ & 0.055 \\
\hline \multicolumn{5}{|l|}{$\operatorname{PLT}\left(10^{3} / \mu \mathrm{L}\right)$} \\
\hline$\geq 10^{5}(\mathrm{n}=164)$ & $1.39(0.71-2.68)$ & 0.338 & & \\
\hline \multicolumn{5}{|l|}{ Albumin (g/dL) } \\
\hline$\geq 3.5(n=173)$ & $0.39(0.22-0.72)$ & 0.002 & & \\
\hline \multicolumn{5}{|l|}{ Myostatin (pg/mL) } \\
\hline$\geq 3,800(n=119)$ & $0.57(0.32-1.04)$ & 0.065 & & \\
\hline \multicolumn{5}{|l|}{ Follistatin (pg/mL) } \\
\hline$\geq 2,100(n=119)$ & $3.54(1.87-6.70)$ & $<0.001$ & $2.21(1.13-4.30)$ & 0.020 \\
\hline \multicolumn{5}{|l|}{ IL-6 (pg/mL) } \\
\hline$\geq 2.5(n=116)$ & $2.04(1.12-3.74)$ & 0.021 & & \\
\hline \multicolumn{5}{|l|}{ MELD score } \\
\hline$\geq 8(n=68)$ & $2.07(1.14-3.75)$ & 0.017 & $2.17(1.17-4.01)$ & 0.013 \\
\hline \multicolumn{5}{|l|}{ ECOG PS } \\
\hline $1,2(n=85)$ & $2.78(1.54-4.99)$ & 0.001 & $1.54(0.83-2.88)$ & 0.174 \\
\hline \multicolumn{5}{|l|}{ TNM stage } \\
\hline Stage II, III, IV & $13.3(4.75-37.22)$ & $<0.001$ & $9.46(3.33-26.9)$ & $<0.001$ \\
\hline \multicolumn{5}{|l|}{ Sarcopenia } \\
\hline Yes $(n=135)$ & $2.33(1.21-4.53)$ & 0.012 & $2.11(1.07-4.17)$ & 0.031 \\
\hline PMI $\left(\mathrm{cm}^{2} / \mathrm{m}^{2}\right)$ & $0.95(0.78-0.17)$ & 0.640 & & \\
\hline
\end{tabular}

HR, hazard ratio; Cl, confidence interval; BMI, body mass index; PY, pack year; AFP, alpha-fetoprotein; PLT, platelet; IL-6, interleukin-6; MELD, model for endstage liver disease; ECOG PS, Eastern Cooperative Oncology Group Performance Status Scale; TNM, tumor-node-metastasis; PMI, psoas muscle index.

the PMI and were associated with a reduced overall survival rate. $^{12}$ In a rat portacaval anastomosis model that reproduces a similar metabolic change to liver cirrhosis, sarcopenia was associated with portosystemic shunting. In this model, sarcopenia was caused by increased myostatin expression, impaired muscle protein synthesis and satellite cell (myocyte precursor) dysfunction, which was reversed by follistatin. ${ }^{26}$

However, in these Korean HCC patients (median age of 59 


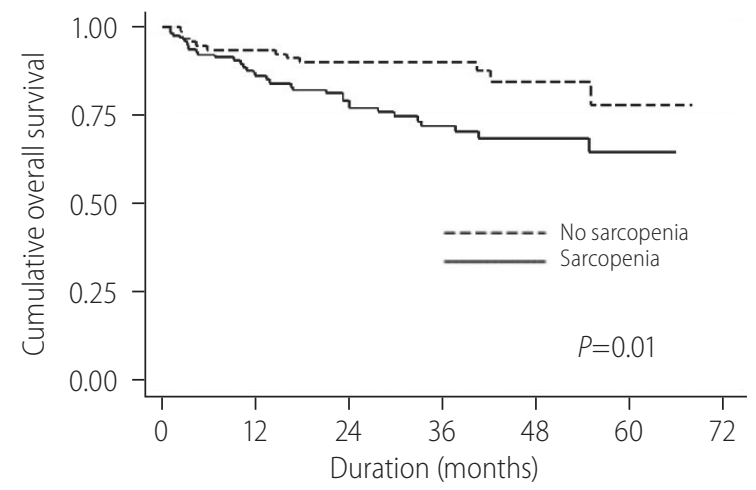

Number at risk

$\begin{array}{llllllll}\text { No sarcopenia } 103 & 81 & 74 & 51 & 22 & 8 & 0\end{array}$

Sarcopenia $\begin{array}{lllllll}135 & 95 & 76 & 47 & 27 & 8 & 0\end{array}$

Figure 4. Overall survival rate in the patients with sarcopenia and nonsarcopenia. In Kaplan-Meier survival analysis, the hepatocellular carcinoma patients with sarcopenia showed a significantly lower survival than the patients with non-sarcopenia.

years; $81 \%$ male; $85 \%$ Child-Pugh class A), the serum myostatin levels showed a positive correlation with the PMI and were not a significant factor for overall survival. The mechanism of this discrepancy between cirrhosis and HCC is not clear, but the development of HCC may change the regulatory pathway of myostatin secretion. In agreement with our results, the serum myostatin level was decreased in various cancer cachexia showing a positive correlation with muscle mass. ${ }^{27,28}$ Cancer is a chronic state of energy malnutrition, which may increase the expression of a splice variant of myostatin leading to decreased bioavailability of myostatin and protection from sarcopenia. ${ }^{29}$

Likewise, in Japanese heart-failure patients $(n=41)$, the serum myostatin levels were significantly lower than in the healthy controls and again showed a positive correlation with muscle mass, suggesting that myostatin may have a compensatory action for maintaining skeletal muscle mass. ${ }^{30}$ In the murine model of cancer cachexia, myostatin expression was upregulated in the muscle of the animals, and blocking of the shared receptor of myostatin and activin, activin type II receptor B, prevented muscle atrophy and increased survival. ${ }^{31}$ However, the regulation of myostatin expression in humans is so complex that further investigation is needed. ${ }^{32}$

Follistatin is a multifunctional regulatory protein, mainly secreted from the liver. The primary function of follistatin is the antagonism effect on the TGF-beta superfamily, including myostatin, activin and BMPs. ${ }^{33-35}$ It is a strong inhibitor of myostatin-mediated sarcopenia, and follistatin-overexpressing transgenic mice exhibit a significant increase in muscle mass. Therefore, follistatin or follistatin mimetics have been tried in preclinical use for treating muscle injury, atrophy or sarcopenia. ${ }^{36}$ Moreover, overexpression of follistatin was found in rodent and human HCC tumors, and experimentally administered follistatin has a role in tumor growth promotion and escaping from the tumor suppressive effect of activin or TGF-beta signaling pathways. ${ }^{37-39}$ TGF-beta signaling showed a tumor suppressive effect in the early stages of carcinogenesis, while it later contributes to tumor progression, showing a contextual regulation in HCC. ${ }^{40}$ Myeloid-derived suppressor cells can produce TGF-beta and IL-10, which lead to immunosuppressive milieu in HCC tissues and the development of the immune escape of HCC cells. ${ }^{41}$ The deregulation of the activin/follistatin system may be important during hepatocarcinogenesis. In this study, the follistatin level increased with the tumor stage, higher AFP levels and tumor size, suggesting an oncogenic role of follistatin overexpression according to the HCC progression. It is supported by several studies showing that follistatin regulates tumor angiogenesis, metastasis, and cell apoptosis. ${ }^{42}$

Moreover, the serum follistatin level was an independent factor for poor survival in the HCC patients, which was compatible with the results of Tomoda et al. ${ }^{42}$ who reported that the serum follistatin level in HCC patients was independently correlated with a poor prognosis. Meanwhile, follistatin serves as a stress responsive protein, having a protective role under oxidative stress, physical stress, and cellular energy deficiency such as prolonged fasting, acute exercise, or solid cancers. Solid cancers including HCC are exposed to glucose deficiency and a hypoxic microenvironment, which results in a compensatory high expression of follistatin, which may be related to our findings. The overwhelming excretion of follistatin may exert a suppression of myostatin, leading to a protective effect on sarcopenia in the HCC patients.

IL-6 mediated signal transducer and activator of transcription 3 activation promote hepatocarcinogenesis, and IL-6 is a determinant of gender disparity for HCC development because estrogen inhibits the release of IL-6 from Kupffer cells, showing a reduced risk of HCC development in women compared to men. ${ }^{43}$ The cellular sources of IL- 6 in HCC are the tumor-associated macrophages and HCC cells. IL- 6 is a marker of inflammation with catabolic effects on muscle proteins, and contributes to the progression of sarcopenia. ${ }^{44,45}$ According to a recent meta-analysis, the serum levels for IL-6 correlates negatively with muscle strength in healthy elderly populations. ${ }^{46}$ Moreover, many studies have consistently reported that IL-6 has an important effect on the development of sarcopenia and cachexia and promoting tumor growth and metastasis in cancer patients including HCC. In this study, the serum levels of IL-6 showed a significant negative correlation with the 
PMI and a progressive increase with increasing cancer stage. Therefore, IL-6 may have a major role in the sarcopenia of HCC patients. Whether therapeutic trials aimed at the reduction of the IL-6 activity, such as endurance exercise or anti-IL- 6 antibodies, may be beneficial for cancer associated sarcopenia in humans need to be studied further. ${ }^{47}$ In the aspect of HCC as the ultimate outcome of liver fibrosis, IL-6 has a profibrogenic role, and myostatin seems to be fibrogenic, ${ }^{48}$ while follistatin may attenuate fibrosis, ${ }^{37}$ which warrants further studies.

There are some limitations in this study. First, it was a single center study with the measurement of only 3 myokines at one single time of the HCC diagnosis. The serum ammonia level was closely linked to the development of sarcopenia, however, the serum ammonia level was not measured in this study. Second, only the amount of muscle but not the function or quality of the muscle was evaluated. It would be interesting to explore the association between muscle strength and the 3 myokines. Third, we determined the optimal cut-offs for defining prognostic sarcopenia from our own cohort; thus, the cut-off may overestimate the prognostic separation. It would be necessary to validate the cutoff values in an independent cohort. Lastly, total skeletal muscle index was not measured, which remains a possible underestimation of mortality risk by using PMI, as shown in cirrhosis patients. ${ }^{49}$ Sarcopenia is already a well-known factor for poor survival in HCC; therefore, our study mainly focused on the simultaneous measurement of the 3 myokines and the PMI in HCC patients for the first time to the best of our knowledge. Moreover, by comparison of those 3 myokines to the age and sex-matched healthy controls, the higher levels of the 3 myokines in HCC patients were clearly demonstrated.

In conclusion, the serum levels of myostatin, follistatin, and IL-6 in association with the PMI in HCC patients were presented. The serum myostatin level was negatively, and the IL-6 level was positively correlated with sarcopenia, while the serum follistatin level showed no correlation. The serum follistatin level was an independent factor for poor survival in HCC patients, which warrants further study.

\section{Authors' contribution}

Conception: Jeong SH, Choi K, Ahn JM, Jang HY

Data collection: Choi K, Jang HY, Chung JW, Hwang SH, Choi YS

Data analysis: Choi K, Jang HY, Chung JW, Jang ES, Choi GH, Kim JW, Jeong SH

Manuscript preparation: Choi K, Jang HY, Jeong SH

\section{Acknowledgements}

This study was supported by an intramural grant (02-2017-041) from the Seoul National University Bundang Hospital.

\section{Conflicts of Interest}

The authors have no conflicts to disclose.

\section{SUPPLEMENTARY MATERIAL}

Supplementary material is available at Clinical and Molecular Hepatology website (http://www.e-cmh.org).

\section{REFERENCES}

1. Landi F, Onder G, Russo A, Liperoti R, Tosato M, Martone AM, et al. Calf circumference, frailty and physical performance among older adults living in the community. Clin Nutr 2014;33:539-544.

2. Mijnarends DM, Meijers JM, Halfens RJ, ter Borg S, Luiking YC, Verlaan $S$, et al. Validity and reliability of tools to measure muscle mass, strength, and physical performance in community-dwelling older people: a systematic review. J Am Med Dir Assoc 2013;14:170-178.

3. Cruz-Jentoft AJ, Baeyens JP, Bauer JM, Boirie Y, Cederholm T, Landi $F$, et al. Sarcopenia: European consensus on definition and diagnosis: report of the European Working Group on sarcopenia in older people. Age Ageing 2010;39:412-423.

4. Beaudart $C$, Reginster JY, Slomian J, Buckinx F, Locquet $M$, Bruyère 0 . Prevalence of sarcopenia: the impact of different diagnostic cut-off limits. J Musculoskelet Neuronal Interact 2014;14:425-431.

5. Zillikens MC, van den Berg JW, Wilson JH, Swart GR. Whole-body and segmental bioelectrical-impedance analysis in patients with cirrhosis of the liver: changes after treatment of ascites. Am J Clin Nutr 1992;55:621-625.

6. Hemmingsson E, Uddén J, Neovius M. No apparent progress in bioelectrical impedance accuracy: validation against metabolic risk and DXA. Obesity (Silver Spring) 2009;17:183-187.

7. Dasarathy S, Merli M. Sarcopenia from mechanism to diagnosis and treatment in liver disease. J Hepatol 2016;65:1232-1244.

8. Pedersen BK. Exercise-induced myokines and their role in chronic diseases. Brain Behav Immun 2011;25:811-816.

9. Pedersen BK. Muscles and their myokines. J Exp Biol 2011;214(Pt 2):337-346.

10. Pedersen $B K$, Akerström TC, Nielsen AR, Fischer CP. Role of myokines in exercise and metabolism. J Appl Physiol (1985) 2007;103:10931098.

11. Halmos T, Suba I. The secretory function of skeletal muscles and its 
role in energy metabolism and utilization. Orv Hetil 2014;155:14691477.

12. Nishikawa $H$, Enomoto $H$, Ishii A, Iwata $Y$, Miyamoto $Y$, Ishii $N$, et al. Elevated serum myostatin level is associated with worse survival in patients with liver cirrhosis. J Cachexia Sarcopenia Muscle 2017;8:915-925.

13. Korean Liver Cancer Study Group (KLCSG); National Cancer Center, Korea (NCC). 2014 Korean Liver Cancer Study Group-National Cancer Center Korea practice guideline for the management of hepatocellular carcinoma. Korean J Radiol 2015;16:465-522.

14. Edge S, Byrd D, Compton C, Fritz A, Greene F, Trotti A. AJCC cancer staging manual. 7th ed. New York: Springer, 2010.

15. Marrero JA, Kulik LM, Sirlin CB, Zhu AX, Finn RS, Abecassis MM, et al. Diagnosis, staging, and management of hepatocellular carcinoma: 2018 practice guidance by the American Association for the Study of Liver Diseases. Hepatology 2018;68:723-750.

16. Dodson RM, Firoozmand A, Hyder O, Tacher V, Cosgrove DP, Bhagat $\mathrm{N}$, et al. Impact of sarcopenia on outcomes following intra-arterial therapy of hepatic malignancies. J Gastrointest Surg 2013;17:21232132.

17. Hamaguchi Y, Kaido T, Okumura S, Kobayashi A, Hammad A, Tamai $Y$, et al. Proposal for new diagnostic criteria for low skeletal muscle mass based on computed tomography imaging in Asian adults. Nutrition 2016;32:1200-1205.

18. Kalafateli M, Mantzoukis K, Choi Yau Y, Mohammad AO, Arora S, Rodrigues $S$, et al. Malnutrition and sarcopenia predict post-liver transplantation outcomes independently of the Model for End-stage Liver Disease score. J Cachexia Sarcopenia Muscle 2017;8:113-121.

19. Peng PD, van Vledder MG, Tsai S, de Jong MC, Makary M, Ng J, et al. Sarcopenia negatively impacts short-term outcomes in patients undergoing hepatic resection for colorectal liver metastasis. HPB (Oxford) 2011;13:439-446.

20. Lausen B, Schumacher M. Maximally selected rank statistics. Biometrics 1992;48:73-85.

21. Lausen B, Sauerbrei W, Schumacher V. Classification and regression trees (CART) used for the exploration of prognostic factors measured on different scales. In: Computational Statistics. Heidelberg: Physica-Verlag, 1994:483-496.

22. Hosoyama T, Yamanouchi K, Nishihara M. Role of serum myostatin during the lactation period. J Reprod Dev 2006;52:469-478.

23. McNally EM. Powerful genes--myostatin regulation of human muscle mass. N Engl J Med 2004;350:2642-2644.

24. McPherron AC, Lawler AM, Lee SJ. Regulation of skeletal muscle mass in mice by a new TGF-beta superfamily member. Nature 1997;387:83-90.

25. Peng LN, Lee WJ, Liu LK, Lin MH, Chen LK. Healthy community-living older men differ from women in associations between myostatin levels and skeletal muscle mass. J Cachexia Sarcopenia Muscle
2018;9:635-642.

26. Dasarathy S, McCullough AJ, Muc S, Schneyer A, Bennett CD, Dodig $M$, et al. Sarcopenia associated with portosystemic shunting is reversed by follistatin. J Hepatol 2011;54:915-921.

27. Loumaye $A$, de Barsy $M$, Nachit $M$, Lause $P$, Frateur $L$, van Maanen $A$, et al. Role of activin A and myostatin in human cancer cachexia. J Clin Endocrinol Metab 2015;100:2030-2038.

28. Breitbart A, Scharf GM, Duncker D, Widera C, Gottlieb J, Vogel A, et al. Highly specific detection of myostatin prodomain by an immunoradiometric sandwich assay in serum of healthy individuals and patients. PLoS One 2013;8:e80454.

29. Jeanplong F, Osepchook CC, Falconer SJ, Smith HK, Bass JJ, McMahon $C D$, et al. Undernutrition regulates the expression of a novel splice variant of myostatin and insulin-like growth factor 1 in ovine skeletal muscle. Domest Anim Endocrinol 2015;52:17-24.

30. Furihata T, Kinugawa S, Fukushima A, Takada S, Homma T, Masaki $Y$, et al. Serum myostatin levels are independently associated with skeletal muscle wasting in patients with heart failure. Int J Cardiol 2016;220:483-487.

31. Zhou X, Wang JL, Lu J, Song Y, Kwak KS, Jiao Q, et al. Reversal of cancer cachexia and muscle wasting by ActRIIB antagonism leads to prolonged survival. Cell 2010;142:531-543.

32. Wagner KR, Fleckenstein JL, Amato AA, Barohn RJ, Bushby K, Escolar DM, et al. A phase I/Itrial of MYO-029 in adult subjects with muscular dystrophy. Ann Neurol 2008;63:561-571.

33. Hansen JS, Rutti S, Arous C, Clemmesen JO, Secher NH, Drescher $A$, et al. Circulating follistatin is liver-derived and regulated by the glucagon-to-insulin ratio. J Clin Endocrinol Metab 2016;101:550560.

34. Zhang L, Liu K, Han B, Xu Z, Gao X. The emerging role of follistatin under stresses and its implications in diseases. Gene 2018;639:111116.

35. McPherron AC. Metabolic functions of myostatin and GDF11. Immunol Endocr Metab Agents Med Chem 2010;10:217-231.

36. Kalinkovich A, Livshits G. Sarcopenia--the search for emerging biomarkers. Ageing Res Rev 2015;22:58-71.

37. Kreidl E, Oztürk D, Metzner T, Berger W, Grusch M. Activins and follistatins: emerging roles in liver physiology and cancer. World J Hepatol 2009;1:17-27.

38. Grusch M, Drucker C, Peter-Vörösmarty B, Erlach N, Lackner A, Losert $A$, et al. Deregulation of the activin/follistatin system in hepatocarcinogenesis. J Hepatol 2006;45:673-680.

39. Rossmanith W, Chabicovsky M, Grasl-Kraupp B, Peter B, Schausberger $\mathrm{E}$, Schulte-Hermann R. Follistatin overexpression in rodent liver tumors: a possible mechanism to overcome activin growth control. Mol Carcinog 2002;35:1-5.

40. Fabregat I, Moreno-Càceres J, Sánchez A, Dooley S, Dewidar B, Giannelli G, et al. TGF- $\beta$ signalling and liver disease. FEBS J 
2016;283:2219-2232.

41. Nishida N, Kudo M. Immune checkpoint blockade for the treatment of human hepatocellular carcinoma. Hepatol Res 2018;48:622-634.

42. Tomoda T, Nouso K, Miyahara K, Kobayashi S, Kinugasa H, Toyosawa J, et al. Prognotic impact of serum follistatin in patients with hepatocellular carcinoma. J Gastroenterol Hepatol 2013;28:13911396.

43. Yang YM, Kim SY, Seki E. Inflammation and liver cancer: Molecular mechanisms and therapeutic targets. Semin Liver Dis 2019;39:2642.

44. Krabbe KS, Pedersen M, Bruunsgaard H. Inflammatory mediators in the elderly. Exp Gerontol 2004;39:687-699.

45. Franceschi C, Campisi J. Chronic inflammation (inflammaging) and its potential contribution to age-associated diseases. J Gerontol A Biol Sci Med Sci 2014;69 Suppl 1:S4-S9.
46. Mikó A, Pótó L, Mátrai P, Hegyi P, Füredi N, Garami A, et al. Gender difference in the effects of interleukin- 6 on grip strength - a systematic review and meta-analysis. BMC Geriatr 2018;18:107.

47. Inácio Pinto N, Carnier J, Oyama LM, Otoch JP, Alcântara PS, Tokeshi $F$, et al. Cancer as a proinflammatory environment: metastasis and cachexia. Mediators Inflamm 2015;2015:791060.

48. Delogu W, Caligiuri A, Provenzano A, Rosso C, Bugianesi E, Coratti $A$, et al. Myostatin regulates the fibrogenic phenotype of hepatic stellate cells via c-jun N-terminal kinase activation. Dig Liver Dis 2019;51:1400-1408.

49. Ebadi M, Wang CW, Lai JC, Dasarathy S, Kappus MR, Dunn MA, et al. Poor performance of psoas muscle index for identification of patients with higher waitlist mortality risk in cirrhosis. J Cachexia Sarcopenia Muscle 2018;9:1053-1062. 